conjugate of $H$ in the equivalent left co-set, and vice versa.

A necessary and sufficient condition that $T$ is simply transitive is that at least one conjugate of $H$ under $G$ has its operators distributed among less than $n-1$ co-sets of $G$ with respect to $H$. In particular, when these operators are distributed among $n-2$ such co-sets $n$ must be even and $T$ must involve a system of imprimitivity composed of $n / 2$ sets of letters. This is also a necessary and sufficient condition that $H$ is invariant under a subgroup of $G$ whose order is exactly $2 h$. If $G$ is simply isomorphic with $T$ and a subgroup of $G$ has its operators distributed among $n-2$ of the co-sets of $G$ with respect to $H$, its order cannot exceed $2 h$, and when it has this order it must involve a subgroup of index 2 which is conjugate with $H$ under $G$. Moreover, $H$ corresponds to a transitive subgroup of degree $n-2$ in $T$.

The University of Illinois

\title{
A NOTE ON TRANSFINITE ORDINALS
}

\section{BY BEN DUSHNIK}

In a supplementary note to an article of theirs, ${ }^{*}$ Alexandroff and Urysohn demonstrated the following theorem.

If to every ordinal $\alpha$ of the second class there corresponds an ordinal $\mu(\alpha)$ such that $\mu(\alpha)<\alpha$, then there exists a non-denumerable set of ordinals of the second class

$$
\alpha_{1}, \alpha_{2}, \cdots, \alpha_{n}, \cdots, \alpha_{\omega}, \cdots, \alpha_{\lambda}, \cdots
$$

such that

$$
\mu\left(\alpha_{1}\right)=\mu\left(\alpha_{2}\right)=\cdots=\mu\left(\alpha_{\lambda}\right) \cdots .
$$

The present note applies a different method to prove the following more general result.

Theorem. Let $\Omega_{\delta}$ be the smallest ordinal whose power is $\boldsymbol{N}_{\delta}$, where $\delta>0$ is a non-limiting ordinal. If to every transfinite ordinal $\alpha<\Omega_{\delta}$ there corresponds an ordinal $\mu(\alpha)$ such that $\mu(\alpha)<\alpha$,

* Mémoire sur les espaces topologiques compacts, Verhandelingen of the Amsterdam Academy, (1), vol. 45, No. 1. 
then there will be a set of power $\boldsymbol{N}_{\delta}$ of transfinite ordinals $<\Omega_{\delta}$ such that the corresponding $\mu(\alpha)$ are all equal.

Proof. Consider, for any transfinite $\alpha<\Omega_{\delta}$, the series

$$
\alpha, \mu(\alpha), \cdots, \mu^{i}(\alpha), \cdots,
$$

where $\mu^{i}(\alpha)$ is the ordinal corresponding to $\mu^{i-1}(\alpha)[i \geqq 2]$. By hypothesis

$$
\alpha>\mu(\alpha)>\mu^{2}(\alpha)>\cdots ;
$$

since every descending series of ordinals is finite, and since $\mu^{i+1}(\alpha)$ is well-defined if $\mu^{i}(\alpha)$ is a transfinite ordinal, there must exist a finite number $m(\alpha)$ such that $\mu^{m(\alpha)}(\alpha)=n(\alpha)$, a finite number.

There is thus associated a definite finite number $n(\alpha)$ with every transfinite ordinal $\alpha<\Omega_{\delta}$. If for every finite $n$ the corresponding set of ordinals $\alpha$ for which $n(\alpha)=n$ has the power $\boldsymbol{N}^{(n)} \leqq \boldsymbol{\aleph}_{\delta-1}$, then the set of all ordinals $<\Omega_{\delta}$ would have the power

$$
\sum_{i=0}^{\infty} \mathbf{N}^{(i)} \leqq \mathbf{N}_{0} \cdot \mathbf{N}_{\delta-1}<\mathbf{N}_{\delta}
$$

which is absurd. Hence there must exist a set $K^{\prime \prime}$ of transfinite ordinals $\alpha<\Omega_{\delta}$, such that the power of $K^{\prime \prime}$ is $\boldsymbol{N}_{\delta}$ and such that if $\alpha$ belongs to $K$, then $\mu^{m(\alpha)}(\alpha)=N$, a fixed finite number. Similarly, there will exist a set $K^{\prime}$ contained in $K^{\prime \prime}$, of the same power as $K^{\prime \prime}$, such that if $\alpha$ belongs to $K^{\prime}$, then $m(\alpha)=M$, a fixed finite number.

The ordinals of the set $K^{\prime}$ having been arranged in a wellordered series (say, of type $\Omega_{\delta}$ ),

$$
\alpha_{1}, \alpha_{2}, \cdots, \alpha_{\omega}, \cdots, \alpha_{\beta}, \cdots,
$$

consider the matrix of $M$ columns in which the row of index $\beta$ contains the elements

$$
\alpha_{\beta}, \mu\left(\alpha_{\beta}\right), \cdots, \mu^{M}\left(\alpha_{\beta}\right),
$$

in the order as given. Since in the first column of the matrix there are $\boldsymbol{\aleph}_{\delta}$ different ordinals, while in the last column the same number $N$ is repeated, there must be a column of index $q<M$ in which appear $\boldsymbol{\aleph}_{\delta}$ distinct ordinals, such that in the $(q+1)$ th column there will appear at most $\aleph_{\delta-1}$ distinct ordi- 
nals. As before, one can obtain from the $q$ th column of the matrix at least one set $K$ of $\boldsymbol{N}_{\delta}$ distinct ordinals $\mu^{q}(\alpha)$ for which all the $\mu^{q+1}(\alpha)$ are identical.

Obviously, this set $K$ is the one whose existence was to be proved.

It may be noted that the Zermelo Axiom has been used freely in the above discussion. Further, it should be mentioned that the method of Alexandroff and Urysohn will also suffice to prove the theorem stated here; however, it is hoped that the method of proof presented here is not without interest.

Finally, the specification in the theorem that $\delta$ be a nonlimiting number is essential, as is indicated by the following example.

Let $\delta=\omega$, the smallest transfinite ordinal, and consider the set of all the transfinite ordinals $\alpha<\Omega_{\omega}$, the power of the set being thus $\boldsymbol{\aleph}_{\omega}$. If $\omega \leqq \alpha<\Omega_{1}$, we put $\mu(\alpha)=1$; in general, for $n \geqq 1$, if $\Omega_{n} \leqq \alpha<\Omega_{n+1}$, we set $\mu(\alpha)=\Omega_{n-1}$, where $\Omega_{0}=\omega$. Except that $\delta$ is now a limiting number, all the other conditions of the theorem are satisfied; yet the conclusion no longer holds. Indeed, $\mu(\alpha)$ is equal to one of the numbers

$$
1, \omega, \Omega_{1}, \Omega_{2}, \cdots, \Omega_{n}, \cdots,
$$

while the set of $\alpha$ 's for which, say, $\mu(\alpha)=\Omega_{n}$ has the power $\boldsymbol{\aleph}_{n+2}<\boldsymbol{\aleph}_{\omega}$.

The University of Michigan 